\title{
Detecting Acceleration for Gait and Crime Scene Analysis
}

\author{
Y.Sun*, J.S. Hare, M.S. Nixon \\ University of Southampton, UK, ys1d14@soton.ac.uk
}

Keywords: Acceleration, Heel strike, Gait, DeepFlow

\begin{abstract}
Identifying criminals from CCTV footage is often a difficult task for crime investigations. The quality of CCTV is often low and criminals can cover their face and wear gloves (to withhold fingerprints) when committing a crime. Gait is the optimal choice in this circumstance since people can be recognised by their walking style, even at a distance with low resolution imagery. The location of the frame when the heel strikes the floor is essential for some gait analyses. We propose a new method to detect heel strikes: by radial acceleration which can also generalise to crime analysis. The frame and position of the heel strikes can be estimated by the quantity and the circle centres of radial acceleration, derived from the optical flow (using DeepFlow). Experimental results show high detection rate on two different gait databases and good robustness under different kinds of noise. We analyse detection of heel strikes to show robustness then we analyse crime scenes to show generalisation capability since violent crime often involves much acceleration. As such, we provide a new basis to a baseline technique in crime scene analysis.
\end{abstract}

\section{Introduction}

Identifying criminals from surveillance videos is often difficult for police because the quality of images is severely affected by illumination and insufficient image resolution. Criminals also cover their faces to avoid recognition which compounds the investigation difficulties. In this case, gait is the optimal biometric technique to recognise criminals.

Gait is a behavioural biometric obtained at a distance from the camera, which is hard to hide or disguise. Since it is not affected by the low quality of images, gait is the most reliable biometric in the criminal investigation when other biometrics are not available. It has been demonstrated previously that gait can be used in criminal investigations either as body measurements [1] or gait measurements [2]. Figure 1 is a CCTV footage of an Australian jewellery shop murder: the target covered his face during the crime and he was recognised by his gait after it was found in the surveillance video that he had come to the jewellery shop earlier that day. In gait analysis, heel strikes are an important and preliminary cue for gait analysis because gait period, step and stride length can be derived accurately by the moment and position of heel strike. It refers to the heel first makes contact with the ground during the stance phase of the walking cycle [3].

We introduce a new method to use acceleration detect the time when a heel strikes the floor and likely crime events.
When the foot is approaching heel strike, its motion status changes from moving forward to making circular motion centred at the heel. The amount of acceleration on the leading foot will dramatically increase when the heel strikes the floor. According to this clue, we can determine the key frame and position of the heel strike precisely. Previous approaches to heel strike detection have used more of the image sequence and have determined the frame which has the most corners [4] and by detection based on the sinusoidal movement of the head and a silhouette accumulator map [5]. In contrast, the new approach uses only three consecutive frames to detect acceleration and thence heel strikes, and further can be generalised to detect crime events which invariably involve acceleration rather than smooth movement.

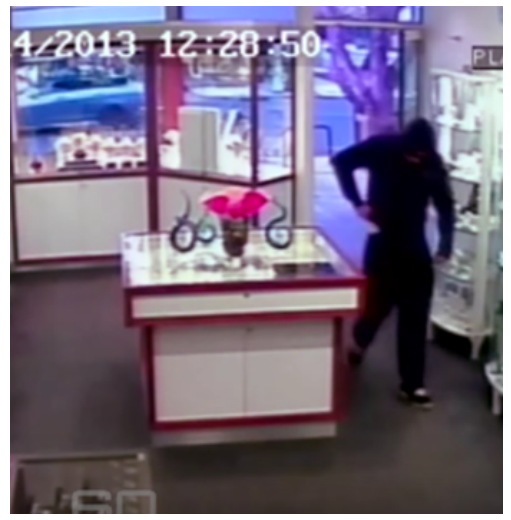

Figure 1: Murderer who was recognised by his gait. ${ }^{1}$

This paper is the first to use acceleration to analyse gait and crime scenes, and we show that this new basis provides a robust and accurate method to automatically describe these basic events. The paper is arranged as follows: Section 2 presents the new algorithm for disambiguating acceleration from optical flow. Section 3 describes each stage of our heel strike detection approach. Experimental results and the robustness of our algorithm are illustrated in Sections 4 and 5, followed by the suggestion for future avenues of research.

\section{Detecting acceleration in computer images}

In past research in motion analysis in computer vision, researchers have only considered the relative movement of objects between consecutive frames. According to the sampling rate of video, the detected displacement can be formed as the 'velocity' of moving objects. There are actually many different types of motion in the real world. There has

\footnotetext{
1 Image is taken from: https://www.youtube.com/watch?v=F1b_apXjjV0\&feature=youtu.be
} 
been no prior study on acceleration, only on velocity or motion.

Acceleration is a vector of describing the magnitude and direction of the change of velocity. The average acceleration is the average rate of change of velocity with respect to the time interval. As with velocity, when the time period approaches zero, it is termed instantaneous acceleration $a$ :

$$
\boldsymbol{a}=\lim _{\Delta t \rightarrow 0} \frac{\Delta v}{\Delta t}=\frac{d v}{d t}
$$

Few objects have linear motion in real-world image sequences so decomposing the resultant acceleration into tangential and radial acceleration is necessary. In a curved motion, the tangential component changes the magnitude of velocity and the direction is located in the tangent line of the trajectory (increasing or decreasing the speed). The radial component (also called centripetal acceleration in a circular motion) changes the direction of velocity and it points to the centre of the curved path (normal to the direction of velocity) [6], as shown in Figure 2. We show how it is possible to decompose acceleration into terms of direction and magnitude.

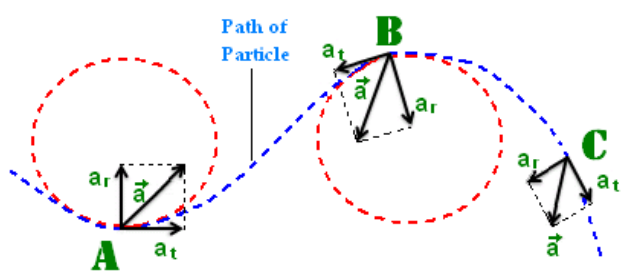

Figure 2: The relationship between resultant acceleration, tangential acceleration and radial acceleration ${ }^{2}$.

At each point, according to the geometry shown in Figure 2, the radial acceleration $\boldsymbol{a}_{r}$ and tangential acceleration $\boldsymbol{a}_{\boldsymbol{t}}$ components can be derived from the resultant acceleration $a$ as:

$$
\boldsymbol{a}_{\boldsymbol{r}}=\sin \theta * \boldsymbol{a}, \boldsymbol{a}_{\boldsymbol{t}}=\cos \theta * \boldsymbol{a}
$$

where $\theta$ is the angle between $\boldsymbol{a}$ and $\boldsymbol{a}_{\boldsymbol{t}}$ and equals the angle between $\boldsymbol{a}$ and $\boldsymbol{v}$ as the direction of velocity is also along the tangent:

$$
\theta=\cos ^{-1} \frac{\boldsymbol{a} \cdot \boldsymbol{v}}{|\boldsymbol{a}||\boldsymbol{v}|}
$$

According to:

$$
a_{r}=v^{2} / r
$$

the radius of the circular motion can be determined by:

$$
\mathbf{r}=\boldsymbol{v}^{2} /(\sin \theta * \boldsymbol{a})
$$

then the centre of radial acceleration that point $(x, y)$ experienced is:

$$
\mathbf{o}=(x, y)-\left(r_{x}, r_{y}\right)
$$

In this work, optical flow is used as the motion detection technique. Optical flow refers to the apparent motion between the observer and the observed object caused by relative motion [7] and it has been steadily developed over 30 years since Horn and Schunck presented the first variational approach in computer vision [8]. Nowadays optical flow estimation methods have achieved a remarkable level of reliability and precision $[9,10,11]$. Among them, DeepFlow

${ }^{2}$ Image is taken from: http://physics.tutorvista.com/motion/tangential-acceleration.html
[12] is a recent technique with excellent performance for large displacement estimation and non-rigid matching. In this paper, the components of resultant acceleration on $x$-axis $a_{u}$ and $y$-axis $a_{v}$ are achieved by differencing consecutive estimates of optical flow detected by DeepFlow:

$$
\begin{aligned}
& a_{u}=u(t+1)-u(t) \\
& a_{v}=v(t+1)-v(t)
\end{aligned}
$$

where $u(t)$ and $v(t)$ denote optical flow components (velocity) of one point in the image along the horizontal and vertical direction at time $t$

The approach to detect acceleration is then to use DeepFlow to determine the estimates of velocity and from them derive acceleration by Equation 7 and determine radial and tangential acceleration by Equation 2 and 3, the centre of radial acceleration from Equation 6.

\section{Heel strike detection}

\subsection{Key Frame Detection}

At heel strike the acceleration on the front foot increases dramatically, due to the disappearance of velocity (deceleration). Also, the striking foot's motion is approximately circular, centred at the heel for a small period of time. Hence, most acceleration on the front foot is radial in nature. Heel strikes can be detected by determining when the number of radial acceleration of the front foot is the maximum. The position of the heel strike is the (circle) centre of radial acceleration.

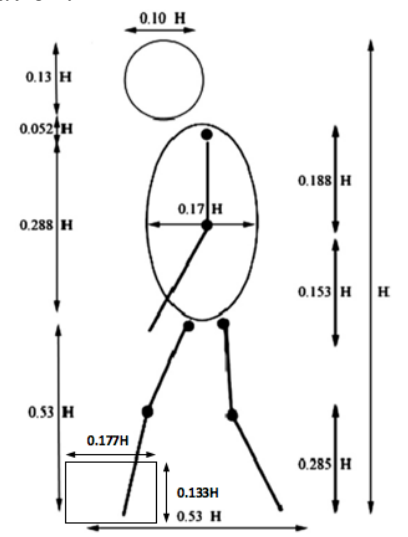

Figure 3: Gait proportions [13]

In implementation, we only consider the radial acceleration in the area we are interested in (the leading foot). The detection area is extracted according to a model of a walking human [13]. Regarding experiments, the area of detection $\boldsymbol{D}$ is defined as a rough area around the leading foot and the size of the area is $0.133 \mathrm{H} \times 0.177 \mathrm{H}$ as illustrated in Figure 3. Only the radial acceleration in this area and points to the heel (below and posterior to the acceleration starting point) will be considered as valid data in our experiments.

\subsection{Heel strike position detection and verification}

When the heel strikes, the front foot is performing circular motion centred at the heel. We assume all the radial 
acceleration in the detection region is caused by a heel strike, then their centres of acceleration should all located at the same position: the heel (ie. the heel strike position).

The radial acceleration estimation algorithm is repeated for all points in the detection region to derive a set of all possible heel strike positions. In order to reduce the effect of noise, the location of the heel strike in frame $t$ is estimated by a weighted sum of radial acceleration circle centres in the detection area:

$$
\mathbf{h e e l}_{x, y}=\sum_{i, j \in \boldsymbol{D}} \alpha(k) * \mathbf{0}_{i, j}(k)
$$

where $\mathbf{O}$ is the set of all detected radial acceleration centres in detection area $D$. The weighting factor $\alpha(k)$ is determined by:

$$
\alpha(k)=n / s
$$

$n$ is the number of radial acceleration centres that are located at the $k$ th position, and $s$ is the number of total detected radial acceleration in the detection region $\boldsymbol{D}$.

\section{Experimental results}

\subsection{Key Frames Detection}

Figure 4 shows a key frame at the detected moment of heel strike. The green square in the silhouette image is the detection region. Figure 5 is the histogram of radial acceleration within a walking sequence. A threshold has been applied to reduce the effect of noise. In the sequence, the radial acceleration appears regularly and noticeably during the sequence, showing the periodicity of gait. However, in frame 64, 78 and 105, some radial acceleration also appears in the frames before or after heel strike. This is caused by the low frame rate. At these moments, the real heel strike occurs between the two consecutive frames.

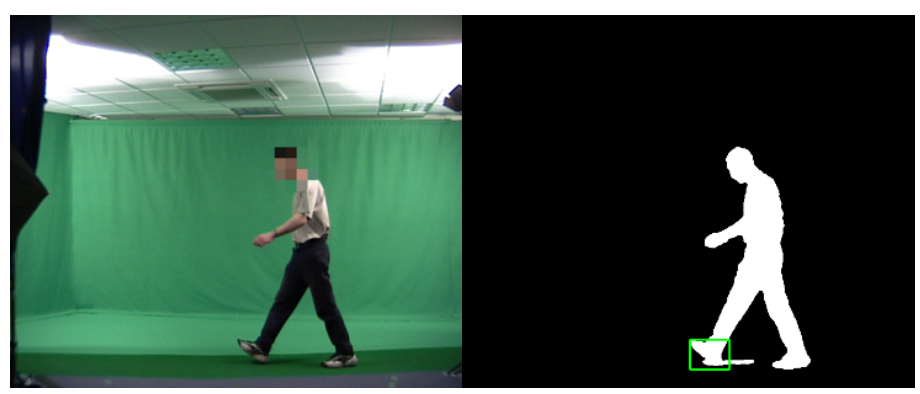

Figure 4: Detecting the region of interest.

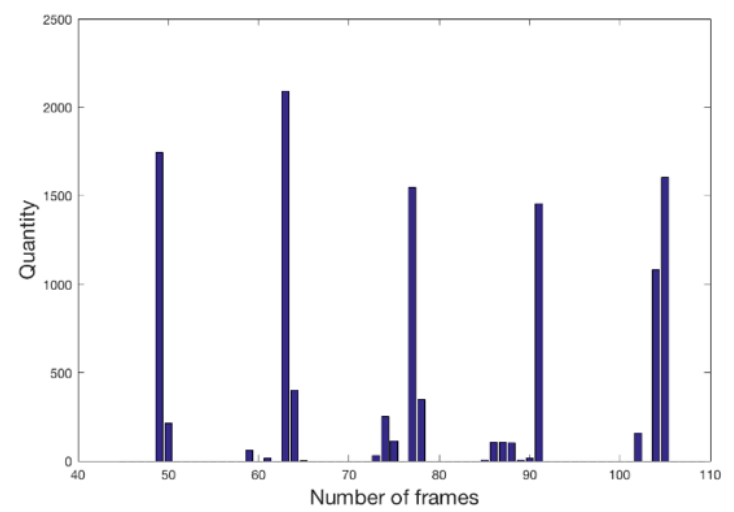

Figure 5: Detected radial acceleration of the leading feet.

\subsection{Heel strike position verification}

The area of interest derived by gait proportions does not locate the leading foot precisely because the shape of the human body changes during the gait cycle. Also, a part of the calf can sometimes be included in the detection region. Moreover, there is also radial acceleration on other areas of the body because the limbs' motion is that of several joined pendulums [3]. The erroneous radial acceleration vectors might also form some invalid heel strike candidates. To obtain accurate heel strike position which estimated by Equation 6, we use detected key frames to verify the heel strike candidates. In other words, the position of the heel strike is only considered to be in the frames in which the radial acceleration on the front foot peaks. If a heel strike occurs between frames, the position is obtained by the weighted coordinates sum according to the amount of radial acceleration in each frame (Equation 8 and 9). Figure 6(a) shows detected candidates of heel strike positions and (b) is the result after being filtered by key frames. The periodicity of gait is evident in the result.

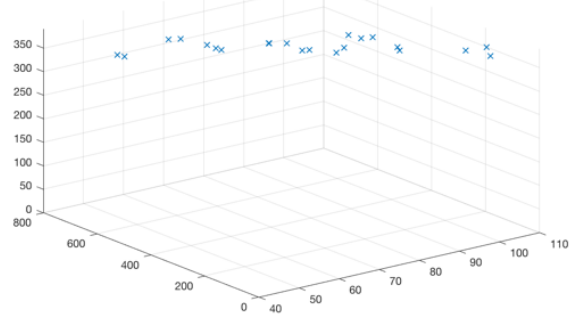

(a) Candidates for heel strikes

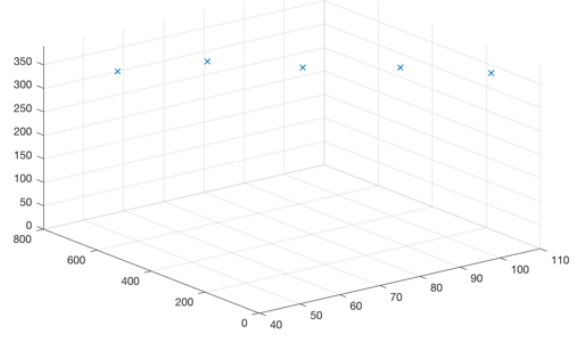

(b) Detected heel strikes (after filtering)

Figure 6: Heel strike verification process.

\begin{tabular}{ll}
\hline Database & Detection rate \\
\hline SOTON & $95.2 \%(254 / 267)$ \\
\hline OU-ISIR & $94.8 \%(369 / 391)$ \\
\hline
\end{tabular}

Table 1: Heel strike detection rates on different databases.

The images in Figure 7 illustrate the detection results on two different databases. These are the indoor SOTON [14] and OU-ISIR [15] gait datasets. Our heel strike detection system performs very well in both of them even if the lighting condition, the angles of view and walking direction are all different. Table 1 shows the outline detection rates of 50 sequences chosen at random from each database compared with the manually labelled ground truth. A distance of horizontal coordinates within \pm 5 pixels is considered as 
successfully detected. We successfully detected 254 out of 267 heel strikes in the SOTON and 369 out of 391 in OUISIR dataset. Compared with the results of a previous study of detecting heel strikes (95.6\% on the SOTON gait database) [5], the detection rate is similar and our approach only requires three consecutive frames. The results in Figure 7(a) also show capability to detect heel strikes in outdoor imagery where the lighting is uncontrolled.
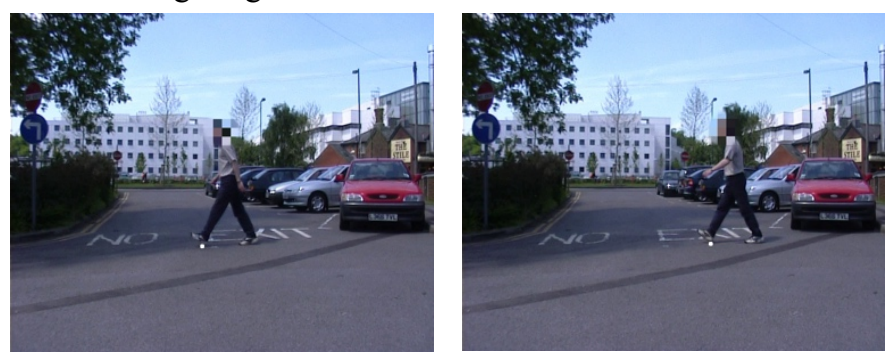

(a) SOTON outdoor images
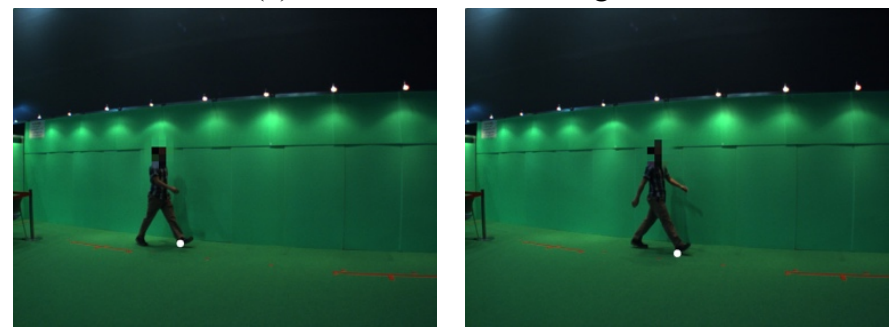

(b) OU-ISIR indoor images
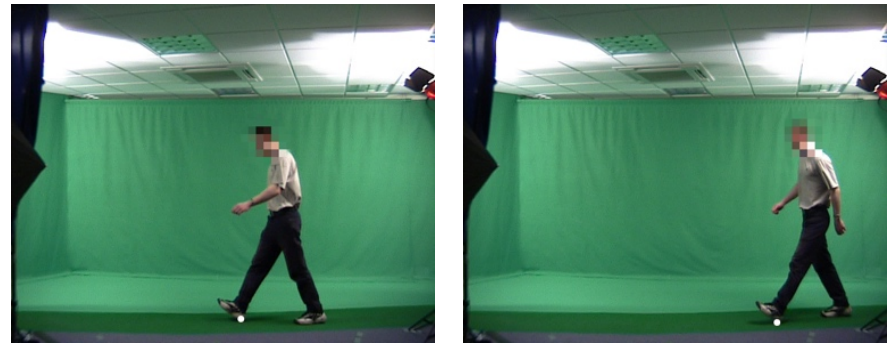

(c) SOTON indoor images

Figure 7: The heel strike detection results of different databases.

\subsection{The noise immunity of heel strike detection approach}

Since the performance of a system under interference is an important issue, we evaluate the robustness of our heel strike detection technique in this section. Three different types of noise that might deteriorate the detection results are added to the original gait sequences: Gaussian zero-mean white noise, occlusion in the detection area and insufficient resolution of the object. These noise reflect some of the anticipated difficulties when applying this new technique to real surveillance videos. Figure 8 illustrates the detection results of the noise at different levels. The results are evaluated by Fscore:

$$
F=2 * \frac{\text { precision } * \text { recall }}{\text { precision }+ \text { recall }}
$$

First Gaussian distributed zero-mean white noise was added to each frame in the sequences. The accuracy of heel strike detection result drops dramatically when the variance increased to 12 and beyond as shown in Figure 9(a). Figure 8(a) shows that the image is quite adversely affected by this level of noise and it is not inconsistent with poor quality surveillance video.

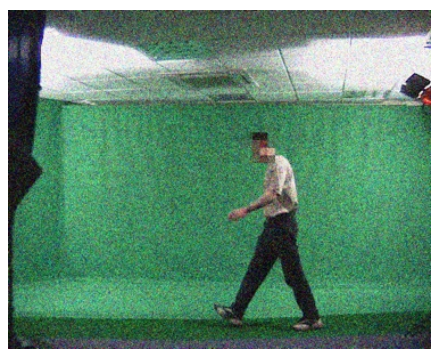

(a) Zero-mean Gaussian white noise $(\sigma=12)$

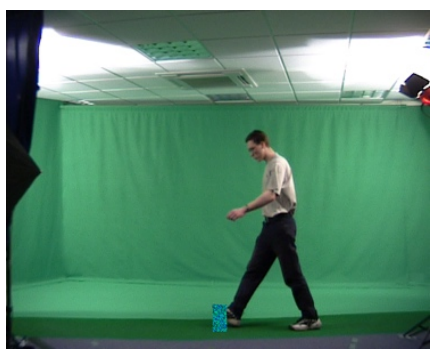

(b) occlusion $(40 \%)$
Figure 8: Different types of noise are incorporated to the original sequence.

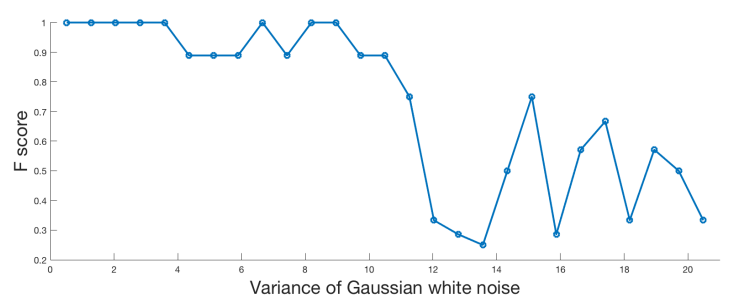

(a) Testing immunity to Gaussian white noise

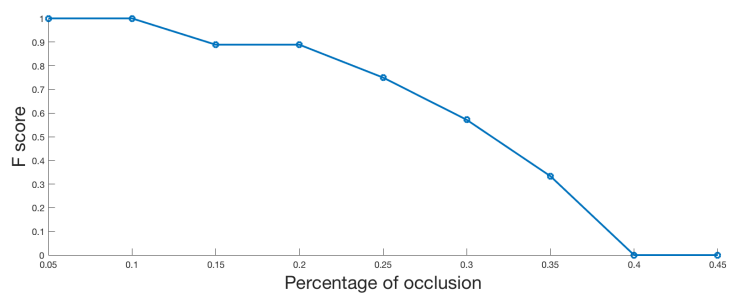

(b) Testing immunity to occlusion

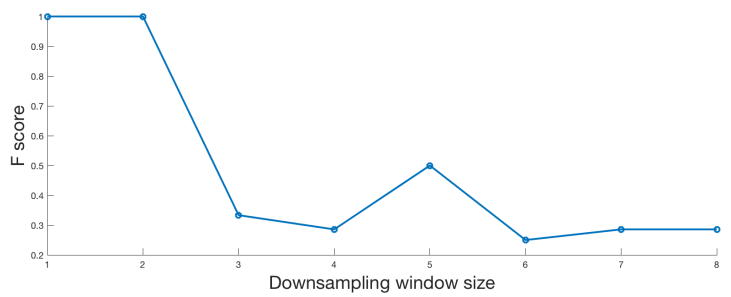

(c) Testing immunity to low resolution

Figure 9: Performance analysis of heel strikes.

Adding occlusion concerns whether the gait information in the real world image sequences is complete or not. In the experiment of testing the immunity of heel strike detecting system to occlusion, we add addition of a random texture to cover the area of interest from toe to heel. The performance on occlusion decreases steadily and our approach totally failed when the detection area are covered over $40 \%$. It is because the pixels on toe travelled the longest when heel strikes but most large radial acceleration located in the toe area are occluded.

Reducing resolution concerns whether resolution of the object might be insufficient in surveillance footage. The original images are downsampled by different window sizes. The Fscore with insufficient resolution decreases below 0.3 when the downsampling window's size increases up to $4 \times 4$ patches 
then the detection results fluctuate at similar level subsequently. The height of the subject is reduced from 350 pixels to 87 approximately when the window size is $4 \times 4$. The most critical issue for evaluating the performance on low resolution images is setting the threshold for the magnitude of radial acceleration. In the experiments, the thresholds are set at the same rate with downsampling but the system still misses most heel strikes. It is the main reason that causes the low F-score while the window size bigger than $2 \times 2$.

Overall, acceleration algorithm shows good robustness in the experiments although there is certain level fluctuation. One of the most important reasons of fluctuation is that the number of heel strike in one gait sequence is low (5 in one sequence). A wrong prediction can make a significant influence on the results. As such the technique appears to be able to tolerate noise, occlusion and resolution effects that are often found in surveillance imagery.

\section{Violent crime analysis using acceleration detection}

Acceleration can also be used as an approach to detect crimes or violence in videos, for example: fighting. When people fight, their body tends to have large acceleration (in many places and with large values) on their body because their arms swing and their feet are kicking. We compare the detected acceleration with the optical flow. Figure 10 shows single images from the acceleration detection results of the surveillance of two episodes in a prison environment. In the left episode there is no fighting and the scene is mundane; in the right a prisoner assaults a guard. In the left episode there is little acceleration detected revealing only the swinging arm of a guard. In comparison there is more optical flow, consistent with more leisurely movement as prisoners receive their visitors. In contrast for the right episode there is considerable detected acceleration in the assault and much less optical flow. When the crimers flee after crime, their body also tends to make more acceleration. As such acceleration appears more suited to the detection of rapid change, consistent with scenes of violence. Thus by detecting acceleration we might be able to determine an approach suited to the detection of violent crime in the future.
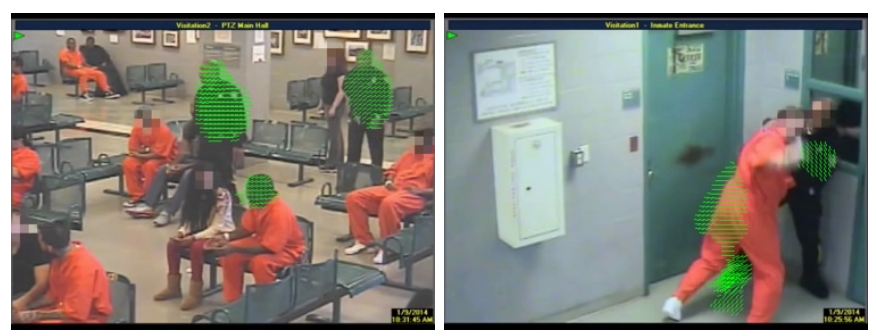

(a) Detecting optical flow
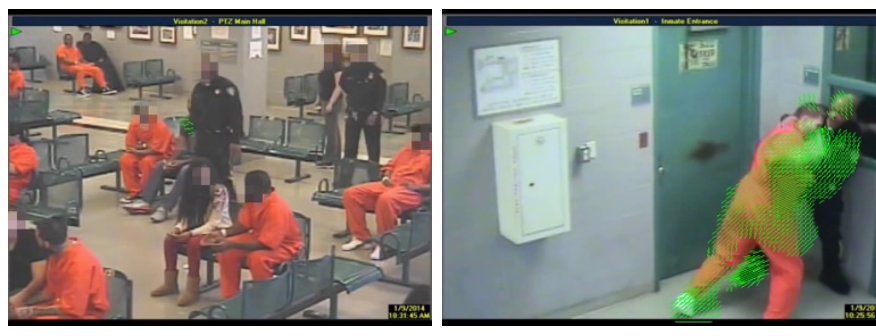

(b) Detecting acceleration

Figure 10: Detecting acceleration and optical flow in an act of violence and in a more relaxed scene. ${ }^{3}$

\section{Conclusions}

This paper is the first study of differentiating from velocity and acceleration in computer images. To understand the motion in the images even more completely, we decomposed acceleration along radial and tangential directions because radial component changes the direction of velocity and tangential component changes the magnitude.

According to the motion feature of heel strike, we use radial acceleration for gait analysis. The change of foot motion status at the instant of heel strike leads to large acceleration in leading foot area. The amount of acceleration arising at heel strike frames give us a conspicuous clue to disambiguating the key frames from the others and the position of heel strike can be estimated by the circle centre of radial components. The leading detection rates are $95.2 \%$ in SOTON Large Database and 94.8\% OU-ISIR Gait Database separately.

Our method also shows a good robustness in performance analysis with respect to noise, occlusion and reduced resolution. Our new approach to acceleration detection and analysis is actually generic and we show that it might have capability to detect acts of violence and we look forward to generalising this capability further.

\section{References}

[1] P. K. Larsen, E. B. Simonsen and N. Lynnerup, "Gait analysis in forensic medicine.," Journal of forensic sciences, vol. 53, no. 5, pp. 1149-1153, 1 Sep 2008.

[2] I. Bouchrika, M. Goffredo, J. Carter and M. Nixon, “On using gait in forensic biometrics," Journal of forensic sciences, vol. 56, no. 4, pp. 882-889, 1 Jun 2011.

[3] D. Cunado, M. S. Nixon and J. N. Carter, "Automatic extraction and description of human gait models for recognition purposes," Computer Vision and Image Understanding, vol. 90, no. 1, pp. 1-41, 30 Apr 2003.

[4] I. Bouchrika and M. S. Nixon, "Gait-based pedestrian detection for automated surveillance.," in International conference on computer vision systems, Bielefeld, 2007.

[5] S.-U. Jung and M. S. Nixon, "Heel strike detection based on human walking movement for surveillance analysis," Pattern Recognition Letters, vol. 34, no. 8, pp. 895-902, 1 Jun 2013.

\footnotetext{
3 Video is taken from https://www.youtube.com/watch?v=RzxS72eCm6c
} 
[6] R. C. Hibbeler, Engineering Mechanics: Dynamics, 13th Edition ed., Pearson, 2015.

[7] J. J. Gibson, The Perception of the Visual World, Cambridge: The Riverside Press, 1950.

[8] B. K. Horn and B. G. Schunck, "Determining optical flow," Artificial intelligence, vol. 17, no. 1-3, pp. 185203, 1 August 1981.

[9] S. Baker, D. Scharstein, J. P. Lewis, S. Roth, M. J. Black and R. Szeliski, "A database and evaluation methodology for optical flow.," International Journal of Computer Vision, vol. 92, no. 1, pp. 1-31, 1 Mar 2011.

[10] D. Sun, S. Roth and M. J. Black, "A quantitative analysis of current practices in optical flow estimation and the principles behind them.," International Journal of Computer Vision, vol. 106, no. 2, pp. 115-137, 1 Jan 2014.

[11] D. Fortun, P. Bouthemy and C. Kervrann, "Optical flow modeling and computation: A survey.," Computer Vision and Image Understanding, vol. 134, pp. 1-21, 31 May 2015.
[12] P. Weinzaepfel, J. Revaud, Z. Harchaoui and C. Schmid, "Deepflow: Large displacement optical flow with deep matching," in In Proceedings of the IEEE International Conference on Computer Vision, 2013.

[13] F. Tafazzoli and R. Safabakhsh, "Model-based human gait recognition using leg and arm movements," Engineering applications of artificial intelligence, vol. 23, no. 8, pp. 1237-1246, 31 Dec 2010.

[14] J. D. Shutler, M. G. Grant, M. S. Nixon and J. N. Carter, "On a large sequence-based human gait database.," Applications and Science in Soft Computing, pp. 339346, 2004.

[15] H. Iwama, M. Okumura, Y. Makihara and Y. Yagi, "The ou-isir gait database comprising the large population dataset and performance evaluation of gait recognition.," IEEE Transactions on Information Forensics and Security, vol. 7, no. 5, pp. 1511-1521, Oct 2012. 